\title{
Comparing the Accuracy and Precision of Multi-echo Combination Methods for Quantitative Susceptibility Mapping Using Laplacian-Based Methods at $3 \mathrm{~T}$
}

Emma Biondetti ${ }^{1,2}$, Anita Karsa ${ }^{2}$, Francesco Grussu ${ }^{3,4}$, Marco Battiston ${ }^{3}$, Marios C.

Yiannakas $^{3}$, David L. Thomas ${ }^{5,6}$, and Karin Shmueli

${ }^{1}$ Institute for Advanced Biomedical Technologies, Department of Neuroscience, Imaging and Clinical Sciences, “D’Annunzio University” of Chieti-Pescara, 66100 Chieti, Italy

${ }^{2}$ Department of Medical Physics and Biomedical Engineering, University College London, London WC1E 6BT, United Kingdom

${ }^{3} \mathrm{NMR}$ Research Unit, Queen Square MS Centre, Department of Neuroinflammation, UCL Queen Square Institute of Neurology, University College London, London WC1N 3BG, United Kingdom

${ }^{4}$ Radiomics Group, Vall d'Hebron Institute of Oncology, Vall d'Hebron Barcelona Hospital Campus, 08035 Barcelona, Spain

${ }^{5}$ Dementia Research Centre, UCL Queen Square Institute of Neurology, University College London, London WC1E 6BT, United Kingdom

${ }^{6}$ Wellcome Centre for Human Neuroimaging, UCL Queen Square Institute of Neurology, University College London, London WC1E 6BT, United Kingdom

\section{Corresponding author}

Emma Biondetti, PhD

Institute for Advanced Biomedical Technologies, Department of Neuroscience, Imaging and Clinical Sciences, “G. D’Annunzio University” of Chieti-Pescara, via Luigi Polacchi 11, 66100 Chieti, Italy

E-mail: emma.biondetti@unich.it

\section{Funding information}

E. Biondetti was supported by the UK Engineering and Physical Sciences Research Council (EPSRC) (award number: 1489882). A. Karsa was supported by the EPSRC-funded UCL Centre for Doctoral Training in Medical Imaging (EP/L016478/1) and the Department of Health's National Institute for Health Research funded Biomedical Research Centre at University College London Hospitals. D. L. Thomas was supported by the UCL Leonard Wolfson Experimental Neurology Centre (PR/ylr/18575). The Queen Square MS Centre, where part of the MRI scans for this work were performed, is supported by grants from the 
UK MS Society and by the National Institute for Health Research University College London Hospitals Biomedical Research Centre. F. Grussu is currently supported by PREdICT, a study at the Vall d'Hebron Institute of Oncology in Barcelona funded by AstraZeneca.

\section{Disclosures}

The authors declare no conflicts: AstraZeneca was not involved in any aspect concerning this work and has not influenced its content or the decision to submit it for publication. 


\begin{abstract}
Purpose: To compare different multi-echo combination methods for MRI quantitative susceptibility mapping (QSM), aiming to elucidate, given the current lack of consensus, how to optimally combine multi-echo gradient-recalled echo (GRE) signal phase information, either before or after applying Laplacian-base methods (LBMs) for phase unwrapping or background field removal.
\end{abstract}

Methods: Multi-echo GRE data were simulated in a numerical head phantom, and multiecho GRE images were acquired at $3 \mathrm{~T}$ in 10 healthy volunteers. To enable image-based estimation of GRE signal noise, 5 volunteers were scanned twice in the same session without repositioning. Five QSM processing pipelines were designed: one applied nonlinear phase fitting over echo times (TEs) before LBMs; two applied LBMs to the TE-dependent phase and then combined multiple TEs via either TE-weighted or signal-to-noise ratio (SNR)-weighted averaging; two calculated TE-dependent susceptibility maps via either multi-step or single-step QSM and then combined multiple TEs via magnitude-weighted averaging. Results from different pipelines were compared using visual inspection; summary statistics of susceptibility in deep gray matter, white matter and venous regions; phase noise propagation maps (error propagation theory); and, in the healthy volunteers, regional fixed bias analysis (Bland-Altman) and regional differences between the means (non-parametric tests).

Results: Nonlinearly fitting the multi-echo phase over TEs before applying LBMs provided the best compromise between regional accuracy/precision and mitigation of phase noise propagation compared to averaging the LBM-processed TE-dependent phase. This result was especially true in high-susceptibility venous regions.

Conclusion: For multi-echo QSM, combining the signal phase by nonlinear fitting before applying LBMs is recommended.

Abstract word count: 250/250 words

Keywords: magnetic resonance imaging, quantitative susceptibility mapping, multi-echo QSM

Word count (body of the text, excluding the title page, abstract, figure captions and references): 4998/5000 


\section{Introduction}

MRI quantitative susceptibility mapping (QSM) aims to determine the underlying spatial distribution of tissue magnetic susceptibility $(\chi)$ from the phase $(\phi)$ of a single-echo or a multi-echo gradient-recalled echo (GRE) sequence. For multi-echo QSM, there is still a lack of consensus on how to combine the information acquired at different echo times (TEs) and when to perform this combination in the processing pipeline.

Generally, $\phi$ measured using a multi-echo GRE acquisition evolves linearly over time:

$$
\phi(\boldsymbol{r}, T E)=\gamma \Delta B_{T o t}(\boldsymbol{r}, T E) T E+\phi_{0}(\boldsymbol{r})
$$

where $\boldsymbol{r}$ is a vector of image space coordinates, $\gamma$ the proton gyromagnetic ratio, $\Delta B_{T o t}$ the $\chi$-induced total field perturbation along the scanner's z-axis, and $\phi_{0}$ the TE-independent phase offset measured at a nominal TE=0 ms.

Based on Equation 1, previous studies using multi-echo GRE protocols and multi-step reconstruction pipelines for brain QSM at $3 \mathrm{~T}$ have combined the signal from multiple echoes by either averaging (1-4) or fitting (5-7) before or after the application of Laplacian-based methods (LBMs). For QSM, LBMs are commonly used to unwrap the phase, i.e., to remove $2 \pi$ phase aliasing over TEs or in space $(8,9)$. LBMs are also commonly used to remove background fields $\left(\Delta B_{B g}\right)$, i.e., slowly varying field variation inside the region of interest (ROI) generated by sources outside the ROI (10). Based on the properties of the Laplacian of the wrapped phase or $\Delta B_{B g}$, LBMs perform nonlinear operations, thus they must be applied carefully to avoid introducing inaccuracies in the GRE phase (11).

For multi-echo combination in QSM, this study focuses on approaches based on weighted averaging (1-4) or complex nonlinear fitting (NLFit) $(5,6)$, which outperform simpler approaches based on unweighted averaging (12) or linear fitting (7).

Multi-echo combination of the phase over time via weighted averaging has been performed using either TE-based weighting factors $(1,2)$ or phase SNR-based weighting factors (3). Notably, TE-weighted averaging (TE-wAvg) only requires temporal unwrapping of the input multi-echo phase and results in a combined total field map $\left(\Delta B_{T o t}\right)$, which still contains $\Delta B_{B g}$ contributions. Instead, SNR-weighted averaging (SNR-wAvg) requires both temporal and spatial unwrapping of the input multi-echo phase, thus resulting in a combined local field map $\left(\Delta B_{L o c}\right)$ free from $\Delta B_{B g}$. Alternatively, a distinct $\chi$ map has been calculated at each TE and then combined by weighted averaging with magnitude-based weighting factors (4). To determine the most accurate multi-echo combination method for QSM, this study aimed to compare the effect of these different approaches on the bias of the estimated tissue $\chi$. Particularly, two different pipelines based on weighted averaging of TE-dependent $\chi$ images were implemented: in one, TE-dependent $\chi$ images were calculated using a multi-step 
procedure similar to NLFit, TE-wAvg, and SNR-wAvg (Susc-wAvg); in the other, TEdependent $\chi$ images were calculated in a single-step using total generalized variation (SuscTGV-wAvg) (13). It was hypothesized that combining multiple TEs by fitting the temporally unwrapped multi-echo phase before applying LBMs for spatial phase unwrapping or $\Delta B_{B g}$ removal (NLFit) gives more accurate $\chi$ estimates than averaging after applying LBMs (TEwAvg, SNR-wAvg, Susc-wAvg, or Susc-TGV-wAvg), because LBMs alter the phase temporal evolution of the phase.

To determine the most precise multi-echo combination method for QSM, this study also aimed to compare the effect of fitting or averaging-based approaches on the propagation of noise from the multi-echo GRE phase into the $\Delta B_{T o t}, \Delta B_{L o c}$ and $\chi$ maps. Previous studies using fitting (6) or SNR-wAvg (3) have calculated expressions for the noise in the total field map $\left(\sigma\left(\Delta B_{T o t}\right)\right)$. For TE-wAvg or SNR-wAvg, expressions for $\sigma\left(\Delta B_{T o t}\right)$ have not been calculated and were therefore derived here. For each multi-echo combination method, expressions for noise propagation from $\Delta B_{T o t}$ to the corresponding $\Delta B_{L o c}$ and $\chi$ images were also calculated here.

Bias and noise propagation characteristics of different multi-echo combination methods were investigated using numerically simulated data and images acquired in vivo, incorporating LBMs for both phase unwrapping and $\Delta B_{B g}$ removal into the QSM processing pipelines.

\section{Theory}

This section presents the expressions for multi-echo combination, noise propagation from the multi-echo signal phase to the $\Delta B_{T o t}, \Delta B_{L o c}$ and $\chi$ maps.

\section{Multi-echo combination}

Multi-echo combination (with $n$ echoes) of the phase over time via weighted averaging has been performed as:

$$
\Delta B(\boldsymbol{r})=\frac{1}{\gamma} \sum_{i=1}^{n} w\left(\boldsymbol{r}, T E_{i}\right) \frac{\phi\left(\boldsymbol{r}, T E_{i}\right)}{T E_{i}}
$$

with TE-based weighting factors (TE-wAvg) equal to $(1,2)$ :

$$
w\left(\boldsymbol{r}, T E_{i}\right)=\frac{T E_{i}}{\sum_{j=1}^{n} T E_{j}}
$$

or phase SNR-based weighting factors (SNR-wAvg) equal to (3):

$$
w\left(\boldsymbol{r}, T E_{i}\right)=\frac{T E_{i} \exp \left(-T E_{i} R_{2}^{*}(\boldsymbol{r})\right)}{\sum_{j=1}^{n} T E_{j} \exp \left(-T E_{j} R_{2}^{*}(\boldsymbol{r})\right)}
$$


TE-wAvg requires temporally unwrapped phase images, because the weights in Equation 3 vary over TEs, whereas SNR-wAvg requires both temporally and spatially unwrapped phase images, because the weights in Equation 4 vary over both TEs and image space.

An alternative multi-echo combination approach has calculated a distinct $\chi$ map at each TE and then applied weighted averaging as (4):

$$
\chi(\boldsymbol{r})=\frac{1}{n} \sum_{i=1}^{n} w\left(\boldsymbol{r}, T E_{i}\right) \chi\left(\boldsymbol{r}, T E_{i}\right)
$$

with weighting factors (Susc-wAvg and Susc-TGV-wAvg):

$$
w\left(\boldsymbol{r}, T E_{i}\right)=\frac{M^{2}\left(\boldsymbol{r}, T E_{i}\right) T E_{i}^{2}}{\sum_{j=1}^{n}\left(M^{2}\left(\boldsymbol{r}, T E_{j}\right) T E_{j}^{2}\right)}
$$

where $M$ denotes the signal magnitude.

Multi-echo combination by fitting the signal phase over time according to Equation 1 enables estimation of both $\Delta B_{T o t}$ and $\phi_{0}$ contributions from the coil-combined phase, whereas combining the phase by averaging according to Equations 2-6 does not enable $\phi_{0}$ estimation. Moreover, multi-echo combination using NLFit does not require unwrapped phase images, thus avoiding the application of LBMs before multi-echo combination.

\section{Noise in the total field map}

\section{Fitting}

Regressing the phase on TE using a weighted linear least square fit enables calculation of a field map $\left(\omega^{F i t}=\gamma \Delta B_{T o t}^{F i t}\right.$ in rad/s) and a phase offset $\left(\phi_{0}^{F i t}\right)$ by minimizing the following weighted residual sum of squares (WRSS):

$$
W R S S(\boldsymbol{r})=\sum_{i=1}^{n} w_{i}\left(\phi\left(\boldsymbol{r}, T E_{i}\right)-\left(\omega^{F i t}(\boldsymbol{r}) T E_{i}+\phi_{0}^{F i t}(\boldsymbol{r})\right)\right)^{2}
$$

Where $w_{i}$ is a weighting factor equal to the inverse variance of the phase, i.e. $w_{i}=$ $1 / \sigma^{2}\left(\phi\left(T E_{i}\right)\right)$ and $n$ is the total number of TEs (14).

The values of $\omega^{\text {Fit }}$ and $\phi_{0}^{\text {Fit }}$ that jointly minimize Equation 7 are calculated by solving a system, where the partial derivatives of WRSS relative to both $\omega^{\text {Fit }}$ and $\phi_{0}^{\text {Fit }}$ are jointly set to zero:

$$
\left\{\begin{array}{l}
\frac{\partial W R S S(\boldsymbol{r})}{\partial \omega^{F i t}}=0 \\
\frac{\partial W R S S(\boldsymbol{r})}{\partial \phi_{0}^{F i t}}=0
\end{array}\right.
$$

Omitting the $\boldsymbol{r}$ dependency (because fitting is performed voxel by voxel) solving the system in Equation 8 yields (6):

$$
\omega^{F i t}=\frac{\sum_{i=1}^{n} w_{i} \sum_{i=1}^{n} w_{i} T E_{i} \phi\left(T E_{i}\right)-\sum_{i=1}^{n} w_{i} \phi\left(T E_{i}\right) \sum_{i=1}^{n} w_{i} T E_{i}}{\sum_{i=1}^{n} w_{i} \sum_{i=1}^{n} w_{i} T E_{i}^{2}-\left(\sum_{i=1}^{n} w_{i} T E_{i}\right)^{2}}
$$


Based on error propagation, the noise in $\Delta B_{T o t}$ calculated using a linear least squares fitting approach (6) is equal to:

$$
\sigma\left(\Delta B_{\text {Tot }}^{\text {Fit }}\right)=\frac{\sqrt{\sum_{i=1}^{n} \frac{1}{\sigma^{2}\left(\phi\left(T E_{i}\right)\right)}}}{\gamma \sqrt{\sum_{i=1}^{n} \frac{1}{\sigma^{2}\left(\phi\left(T E_{i}\right)\right)} \sum_{i=1}^{n} \frac{T E_{i}^{2}}{\sigma^{2}\left(\phi\left(T E_{i}\right)\right)}-\left(\sum_{i=1}^{n} \frac{T E_{i}}{\sigma^{2}\left(\phi\left(T E_{i}\right)\right)}\right)^{2}}}
$$

Equation 10 also holds true for nonlinear least squares fitting (5), thus $\sigma\left(\Delta B_{\text {Tot }}^{\text {NLFit }}\right)=$ $\sigma\left(\Delta B_{\text {Tot }}^{\text {Fit }}\right)$.

\section{Weighted averaging}

Based on Equations 2-4, $\Delta B_{\text {Tot }}$ calculated using TE-weighted averaging and SNR-weighted averaging respectively have variances equal to:

$$
\begin{gathered}
\sigma^{2}\left(\Delta B_{T o t}^{T E-w A v g}\right)=\sigma^{2}\left(\frac{\sum_{i=1}^{n} \phi\left(T E_{i}\right)}{\gamma \sum_{i=1}^{n} T E_{i}}\right) \\
\sigma^{2}\left(\Delta B_{T o t}^{S N R-w A v g}\right)=\sigma^{2}\left(\frac{1}{n \gamma} \sum_{i=1}^{n} \frac{T E_{i} \exp \left(-T E_{i} R_{2}^{*}\right)}{\sum_{j=1}^{n} T E_{j} \exp \left(-T E_{j} R_{2}^{*}\right)} \frac{\phi\left(T E_{i}\right)}{T E_{i}}\right)
\end{gathered}
$$

Assuming that noise in the single-echo phase is temporally uncorrelated and based on error propagation, the noise in $\Delta B_{\text {Tot }}$ calculated using TE-weighted or SNR-weighted averaging is respectively equal to:

$$
\begin{gathered}
\sigma\left(\Delta B_{T o t}^{T E-w A v g}\right)=\frac{\sum_{i=1}^{n} \sigma\left(\phi\left(T E_{i}\right)\right)}{\gamma \sum_{i=1}^{n} T E_{i}} \\
\sigma\left(\Delta B_{T o t}^{S N R-w A v g}\right)=\frac{1}{\gamma} \sum_{i=1}^{n} \frac{\exp \left(-T E_{i} R_{2}^{*}\right)}{\sum_{j=1}^{n} T E_{j} \exp \left(-T E_{j} R_{2}^{*}\right)} \sigma\left(\phi\left(T E_{i}\right)\right)
\end{gathered}
$$

Similar to Equation 10, Equations 13 and 14 omit the $\boldsymbol{r}$ dependency, because they combine multi-echo phase in a voxel-wise fashion.

\section{Noise in the local field map}

For all pipelines, based on error propagation and the orthogonality (15) of $\Delta B_{L o c}$ and the $\Delta B_{B g}$ in the ROI, e.g., the brain:

$$
\sigma\left(\Delta B_{L o c}\right)=\sqrt{\sigma^{2}\left(\Delta B_{T o t}\right)-\sigma^{2}\left(\Delta B_{B g}\right)} \Leftrightarrow \sigma\left(\Delta B_{L o c}\right) \leq \sigma\left(\Delta B_{T o t}\right)
$$

where the $\Leftrightarrow$ symbol denotes an "if and only if" relationship. Therefore, in a worst-case scenario, the noise affecting $\Delta B_{L o c}$ equals the noise affecting the corresponding $\Delta B_{T o t}$.

\section{Noise in the susceptibility map}

The noise in the susceptibility map $\sigma(\chi)$ is calculated based on (16):

$$
\chi(\boldsymbol{r})=d^{-1}(\boldsymbol{r}) \star \Delta B_{L o c}(\boldsymbol{r})
$$


where $d$ is the magnetic dipole and $\star$ denotes a spatially-dependent convolution operation. Thus, based on spatial error propagation, $\sigma(\chi)$ is (see Supporting Information):

$$
\sigma(\chi(\boldsymbol{r}))=\frac{1}{B_{0}} \sqrt{\tilde{d}^{-2}(\boldsymbol{r}) \star \sigma^{2}\left(\Delta B_{L o c}(\boldsymbol{r})\right)}
$$

When the regularized inverse dipole function $\tilde{d}^{-1}$ can be derived analytically and independently of $\chi$ or $\Delta B_{L o c}$, e.g., in thresholded k-space division (TKD) or Tikhonovregularized minimal norm solution $(6,16,17)$, Equation 17 can be solved as:

$$
\sigma(\chi(\boldsymbol{r}))=\frac{1}{B_{0}} \sqrt{F T^{-1}\left(\widetilde{D}^{-2}(\boldsymbol{k}) F T\left(\sigma^{2}\left(\Delta B_{L o c}(\boldsymbol{r})\right)\right)\right)}
$$

where $F T$ and $F T^{-1}$ respectively denote the direct and inverse Fourier transforms, $\boldsymbol{k}$ denotes k-space coordinates, and $\widetilde{D}^{-1}$ the magnetic dipole in k-space.

\section{Weighted averaging}

For weighted averaging of TE-dependent $\chi$ (Equations 5 and 6), based on phase error propagation over time and Equation 15, $\sigma\left(\Delta B_{L o c}\left(T E_{i}\right)\right)$ equals:

$$
\sigma\left(\Delta B_{L o c}\left(T E_{i}\right)\right)=\frac{\sigma\left(\phi\left(T E_{i}\right)\right)}{\gamma T E_{i}}
$$

Based on Equations 5, 6, 18 and 19, $\sigma\left(\chi^{\text {Susc-wAvg }}\right)$ is the square root of (see Supporting Information):

$$
\begin{aligned}
\sigma^{2}\left(\chi^{\text {Susc-wAvg }}\right) & = \\
& =\frac{1}{\left(B_{0} \gamma n\right)^{2}} \sum_{i=1}^{n} \chi^{2}\left(T E_{i}\right)\left(\frac{4 M^{2}\left(T E_{i}\right) T E_{i}^{4} \sigma^{2}\left(M\left(T E_{i}\right)\right)}{\sum_{j=1}^{n}\left(M^{2}\left(T E_{j}\right) T E_{j}^{2}\right)^{2}}\right. \\
& +\frac{M^{4}\left(T E_{i}\right) T E_{i}^{4}}{\sum_{j=1}^{n}\left(M^{2}\left(T E_{j}\right) T E_{j}^{2}\right)^{4}} \sum_{j=1}^{n} 4 M^{2}\left(T E_{j}\right) T E_{j}^{4} \sigma^{2}\left(M\left(T E_{j}\right)\right) \\
& \left.-\frac{2 M^{2}\left(T E_{i}\right) T E_{i}^{2}}{\left(\sum_{j=1}^{n} M^{2}\left(T E_{j}\right) T E_{j}^{2}\right)^{3}} 4 M^{2}\left(T E_{i}\right) T E_{i}^{4} \sigma^{2}\left(M\left(T E_{i}\right)\right)\right) \\
& +\left(\frac{M^{2}\left(T E_{i}\right) T E_{i}^{2}}{\sum_{j=1}^{n} M^{2}\left(T E_{j}\right) T E_{j}^{2}}\right)^{2} \sigma^{2}\left(\chi\left(T E_{i}\right)\right)
\end{aligned}
$$

Notably, Equation 20 only applies to the Susc-wAvg pipeline, as it requires analytically describing $\sigma(\chi)$ (Equation 18). In the Susc-TGV-wAvg pipeline, such an equation could not be calculated, because the total generalized variation (TGV) method estimates $\chi$ iteratively (13). 


\section{Methods}

When not otherwise stated, image analysis was performed using Matlab (R2017b, The MathWorks, Natick, MA) and statistical analysis using Stata (R15, StataCorp LP, College Station, TX). A preliminary version of this study was presented at the 2016 annual meeting of the International Society for Magnetic Resonance in Medicine (18).

\section{In vivo data acquisition}

Multi-echo 3D GRE imaging of ten healthy volunteers (average age/age range: 26/22-30 years, five females) was performed in two centers (University College London Hospital and Queen Square Multiple Sclerosis Centre, University College London) equipped with the same 3 T MRI system (Philips Achieva, Philips Healthcare, Best, NL; 32-channel head coil). Five subjects were acquired in each center. All the volunteers provided written informed consent and the local research ethics committees approved the experimental sessions. Images were acquired using a transverse orientation, field of view $=240 \times 240 \times 144 \mathrm{~mm}^{3}$, voxel size $=1 \mathrm{~mm}^{3}$ isotropic, flip angle $=20$ degrees, repetition time $=29 \mathrm{~ms}$, five evenly spaced echoes $\left(\mathrm{TE}_{1} / \mathrm{TE}\right.$ spacing=3/5.4 ms), bandwidth=270 Hz/pixel, SENSE (19) along the first/second phase encoding directions=2/1.5, flyback gradients along the frequency encoding (FE) direction, and without flow compensating gradients. To enable the imagebased calculation of SNRs in the magnitude and phase images, five subjects were scanned twice within the same session without repositioning.

\section{Data simulation from a numerical head phantom}

To ensure the availability of ground-truth $\chi$ values against which to test the accuracy of QSM pipelines, a piece-wise constant Zubal numerical head phantom (20) was used to generate a simulated noisy multi-echo complex data set as previously described (11). The resulting phantom had matrix size $=256 \times 256 \times 128$ voxels $^{3}$, resolution $=1.5 \mathrm{~mm}$ isotropic, and the following regions of interest (ROls): the caudate nucleus $(\mathrm{CN})$, globus pallidus (GP), putamen (PU), thalamus (TH), superior sagittal sinus (SSS), grey and white matter (GM and WM) and cerebrospinal fluid (CSF). Compared to our previous study (11), the numerical phantom was updated to also have realistic standard deviations (SDs) for $\chi$, the proton density $\left(M_{0}\right)$ and the transverse relaxation rate $\left(T_{2}^{*}\right)$ in each ROI (see Supporting Information).

\section{Data preprocessing}

A brain mask was calculated for each subject by applying the FSL Brain Extraction Tool (BET) $(21,22)$ with robust brain center estimation (threshold $=0.3$ ) to the magnitude image at 
the longest TE. The mask was based on the longest TE to account for the greater amount of signal dropout near regions of high- $\chi$ gradients compared to shorter TEs.

A whole-brain mask for the Zubal phantom was calculated by applying FSL BET with robust brain center estimation (threshold $=0.5$ ) to the $T_{2}^{*}$ map of the numerical phantom (11).

\section{Processing pipelines for QSM}

Five distinct processing pipelines (Figure 1) were applied to both the numerically simulated data and the healthy volunteer data. Three of these pipelines (NLFit, TE-wAvg, and SNRwAvg) combined the phase across TEs at different stages before performing the $\Delta B_{L o c}$-to- $\chi$ inversion. The two other pipelines (Susc-wAvg and Susc-TGV-wAvg) first calculated a distinct $\chi$ map at each TE and then combined the $\chi$ maps. The following paragraphs describe each processing pipeline in detail.

The NLFit processing pipeline (11) first combined the complex GRE signal by nonlinear fitting over TEs (5) using the Cornell QSM software package's Fit_ppm_complex function (http://weill.cornell.edu/mri/pages/qsm.html). It then applied simultaneous phase unwrapping and $\Delta B_{B g}$ removal using Sophisticated Harmonic Artifact Reduction for Phase data (SHARP) (23), which is a direct solver of the discretized Poisson equation (23).

SHARP was chosen because it has been widely used in the literature on QSM and is both robust and numerically efficient (10). In addition, in our recent study comparing multi-echo and TE-dependent QSM, a multi-echo pipeline incorporating SHARP gave highly accurate multi-echo QSM values (11). As in this previous study (11), SHARP was implemented using the minimum-size 3-voxel isotropic 3D Laplacian kernel (23), and it was applied using a threshold for truncated singular value decomposition (tSVD) equal to 0.05 and a brain mask with a 2-voxel (numerical phantom) or 4-voxel erosion (healthy volunteers).

The TE-wAvg processing pipeline first applied Laplacian unwrapping to the phase at each TE using a threshold for tSVD equal to $10^{-10}$ (i.e., the default value in the Cornell QSM software package). Second, TE-wAvg calculated $\Delta B_{\text {Tot }}$ by averaging the unwrapped phase according to Equation 2 with the TE-based weights in Equation $3(1,2)$. Then, it calculated $\Delta B_{L o c}$ by applying SHARP with the same parameters as in NLFit and removing residual phase offsets by applying SHARP with variable kernel size (V-SHARP) with an initial kernel radius of approximately $40 \mathrm{~mm}$ and a step size/final kernel radius of 1 voxel $(24,25)$. The SNR-wAvg processing pipeline first applied simultaneous phase unwrapping and $\Delta B_{B g}$ removal to the phase at each TE using SHARP with the same parameters as in NLFit. It then calculated $\Delta B_{L o c}$ by averaging the unwrapped phase according to Equation 2 with the SNR-based weights in Equation 4 (3) and removing residual phase offsets by applying VSHARP as in TE-wAvg. 
In these three pipelines, $\Delta B_{L o c}$-to- $\chi$ inversion was performed using Tikhonov regularization with correction for susceptibility underestimation and using the L-curve method to determine the optimal value for the regularization parameter $(6,23,26)$. This inversion method was chosen because it is computationally efficient and substantially reduces streaking artifacts relative to the truncated k-space division method (27).

The Susc-wAvg processing pipeline calculated a distinct QSM image at each TE by applying simultaneous phase unwrapping and $\Delta B_{B g}$ removal using SHARP as in NLFit, removing residual phase offsets by applying V-SHARP as in TE-wAvg and SNR-wAvg, and performing the $\Delta B_{L o c}$-to- $\chi$ inversion using Tikhonov regularization as in NLFit, TE-wAvg and SNR-wAvg. This pipeline then calculated a combined QSM image by applying Equation 5 with the magnitude-based weights in Equation 6 (4).

The Susc-TGV-wAvg processing pipeline applied one-step TGV (13) to the phase at each TE, and then calculated a combined QSM image as in Susc-wAvg. The TGV method was chosen because it avoids stair-casing artifacts in the resulting QSM image while correctly preserving structural borders (13). Moreover, in our recent study comparing multi-echo and TE-dependent QSM, TGV provided highly accurate TE-dependent QSM images (11). TGV was implemented using Singularity (https://github.com/CAlsr/qsm; Sylabs, Albany, CA) and the default parameter values $\left(\alpha_{1}, \alpha_{0}\right)=(0.0015,0.005)$, which are optimal for medical imaging applications (13).

\section{ROI segmentation in the healthy volunteer images}

Regional $\chi$ values were compared between the simulated and in vivo data similar to our previous study comparing multi-echo and TE-dependent QSM (11). To this aim, ROls similar to the numerical phantom were segmented in vivo: the CN, GP, PU, TH, posterior corona radiata (PCR) as a WM ROI and the straight sinus (StrS) as a venous $\mathrm{ROI}$. In brief, for each subject, the CN, GP, PU, TH and PCR were segmented based on the Eve $\chi$ atlas (28), whose GRE magnitude image was aligned to each subject's fifth-echo magnitude image using NiftyReg $(29,30)\left(\mathrm{TE}_{\mathrm{Eve}} / \mathrm{TE}_{5}=24 / 24.6 \mathrm{~ms}\right)$. The ITK-SNAP active contour segmentation tool (31) was used to segment the StrS in each subject based on the fifth-echo magnitude image, which presented the best contrast between the StrS and the surrounding brain tissue.

\section{Quantitative evaluation of the measured $\chi$}

In the numerical phantom simulations, the performance of each QSM pipeline relative to the ground truth was visually assessed by calculating a difference image between the corresponding $\chi$ map and the $\chi^{\text {True }}$. In the volunteers, owing to the lack of a ground truth, 
similar difference images were calculated relative to $\chi^{\text {NLFit }}$, because the NLFit pipeline provided the lowest regional susceptibility bias in the numerical phantom simulations (see the Results section).

In the numerical phantom simulations, the means and SDs of $\chi$ were calculated for each pipeline in each phantom ROI with $\chi^{\text {True }} \neq 0$, i.e., the CN, GP, PU, TH, SSS and WM. The root mean squared errors (RMSEs) of $\chi$ relative to $\chi^{\text {True }}$ were also calculated throughout the brain volume as previously described (11).

In the volunteers, the means and SDs of $\chi$ were calculated in each ROI and for each processing pipeline and compared against $\chi$ values in subjects of a similar age from the literature. In the volunteers, the RMSEs could not be calculated because of the lack of a ground truth.

For visualization purposes in the healthy volunteers, if it was possible to pool the regional means and SDs of $\chi$ across subjects (i.e., all intrasubject SDs of $\chi$ were larger than the intersubject SD of $\chi$ ), the pooled averages and SDs were calculated as previously described (11).

\section{Noise propagation maps}

Only the healthy volunteers scanned twice were considered for this analysis. To enable image SNR calculation, in one healthy volunteer, five 20x20-voxel ROls were drawn on a sagittal slice of the first-echo magnitude image (32), including both the GM and WM and taking care to exclude regions with artifacts induced by SENSE, motion or flow. All five ROls were then rigidly aligned with the first-echo magnitude image of the four other volunteers using NiftyReg (30).

In each subject, ROI-based magnitude $\left(M_{R O I}\right)$, magnitude noise $\left(\sigma_{R O I}(M)\right)$ and phase noise values $\left(\sigma_{R O I}(\phi)\right)$ were calculated based on the SNR difference method (33):

$$
\begin{array}{cc}
M_{R O I}(T E, \boldsymbol{r})=\frac{1}{2} \operatorname{mean}\left(M_{1}(T E, \boldsymbol{r})+M(T E, \boldsymbol{r})\right), & \boldsymbol{r} \in R O I \\
\sigma_{R O I}(M(T E, \boldsymbol{r}))=\sqrt{\frac{R}{2}} S D\left(M_{1}(T E, \boldsymbol{r})-M_{2}(T E, \boldsymbol{r})\right), & \boldsymbol{r} \in R O I \\
\sigma_{R O I}(\phi(T E, \boldsymbol{r}))=\sqrt{\frac{R}{2}} S D\left(\phi_{1}(T E, \boldsymbol{r})-\phi_{2}(T E, \boldsymbol{r})\right), & \boldsymbol{r} \in R O I
\end{array}
$$

Here, $M_{1} / \phi_{1}$ and $M_{2} / \phi_{2}$ respectively denote the magnitude/phase images from the first and second scan, $R=3$ is the 2D SENSE factor calculated by multiplying the SENSE factors applied along the two phase encoding directions (34). The values calculated based on 
Equations 19-21 were averaged across the five ROls, to calculate summary values of magnitude, magnitude noise and phase noise at each TE.

For both the numerical phantom simulations and the healthy volunteers, a $\sigma(\phi)$ map was calculated at each TE as $(14,35)$ :

$$
\sigma(\phi(T E, \boldsymbol{r}))=c(T E) \frac{1}{S N R(T E, \boldsymbol{r})}=c(T E) \frac{\sigma_{R O I}(A(T E))}{M(T E, \boldsymbol{r})}
$$

where $c(T E)$ was a constant equal to 1 (by definition) in the numerical phantom simulations and equal to:

$$
c(T E)=\frac{\sigma_{R O I}(T E)}{\frac{1}{S N R_{R O I}(M)}}=\frac{\sigma_{R O I}(\phi(T E)) \sigma_{R O I}(M(T E))}{M(T E)}
$$

in the healthy volunteers. Notably, calculating phase noise analytically as in Equation 24 enabled the direct comparison of noise propagation between numerical simulations and data acquired in vivo.

For each of the NLFit, TE-wAvg and SNR-wAvg processing pipelines, the $\sigma\left(\Delta B_{T o t}\right)$ map was calculated based on the multi-echo $\sigma(\phi)$ maps according to Equations 10,13 and 14, respectively. Then, the $\sigma(\chi)$ map was calculated based on the corresponding $\sigma\left(\Delta B_{T o t}\right)$ map (Equation 18) with $B_{0}=3 \mathrm{~T}$ and the Tikhonov-regularized inverse magnetic dipole with regularization parameter $\alpha(6)$ :

$$
\widetilde{D}^{-2}(\boldsymbol{k} ; \alpha)=\frac{D(\boldsymbol{k})}{D(\boldsymbol{k})^{2}+\alpha}
$$

and correction for $\chi$ underestimation (23). For the Susc-wAvg pipeline, the $\sigma(\chi)$ map was calculated based on Equation 20. Notably, as explained in the Theory section, $\sigma(\chi)$ maps were not calculated for the Susc-TGV-wAvg pipeline, because an analytical expression for $\sigma(\chi)$ could not be derived using the TGV method.

To compare $\sigma(\chi)$ maps across pipelines, a line profile was traced in the same location of all $\sigma(\chi)$ images and the $\sigma(\chi)$ value of each voxel along this profile was plotted. To compare the noise intensity and its variability between processing pipelines, the mean and SD of this representative line profile were also calculated.

\section{Statistical analysis}

Statistical analyses were performed based on the healthy subject data. For each $\mathrm{ROI}$ and each pair of pipelines, potential symmetric biases between distinct processing pipelines were investigated using Bland-Altman analysis of the average $\chi$. For each ROI, statistically significant differences between pipelines were tested by considering the corresponding distributions of average $\chi$ values across subjects. To assess whether to apply parametric paired t-tests or nonparametric sign tests, the normal distribution of the differences between 13 
paired $\chi$ values was assessed using the Shapiro-Wilk test. All statistical tests were two-tailed and an uncorrected $P$-value of 0.05 or less was considered significant.

\section{Results}

\section{Pooling of $\chi$ measurements}

For each $\mathrm{ROI}$ and each processing pipeline, all intrasubject SDs of $\chi$ were larger than the intersubject SD. Thus, pooled means and SDs were calculated (11).

\section{Performance of pipelines for multi-echo QSM}

In the numerical phantom, Figure 2 shows the QSM images calculated by each processing pipeline for multi-echo QSM $(A-L)$, their difference relative to the ground truth $(M-V)$, and the RMSEs of $\chi$ throughout the brain volume (bottom row). Figure $3 \mathrm{~A}$ shows the means and SDs of $\chi$ calculated using each processing pipeline in each $\mathrm{ROI}$.

For the numerical phantom simulations, the error between the calculated and ground-truth $\chi$ appeared fairly similar for all processing pipelines, although slightly larger SDs were always observed for the NLFit pipeline (Figures 2M-V and Figure 2A). The SSS, which was the highest $\chi$ structure $\left(\chi^{\text {True }}=0.30 \pm 0.06 \mathrm{ppm}\right)$, showed the largest susceptibility errors for all the processing pipelines (arrowheads in Figures 2G-L, R-V and Figure 3A).

For one representative volunteer, Figures $4 \mathrm{~A}-\mathrm{J}$ show the susceptibility images calculated using each processing pipeline for multi-echo QSM. Additionally, Figures 4K-L show the differences between each QSM image relative to the $\chi^{\text {NLFit }}$ image. For each $\mathrm{ROI}$ in the healthy volunteers, Figure 3B shows the pooled means and SDs of $\chi$ calculated using each processing pipeline. In the volunteers, susceptibility differences between processing pipelines were most prominent in the StrS (arrowheads in Figures 4F-J, O-R). In general, the average $\chi$ measured in the deep-GM ROls and in the PCR (Figure 3B) had values within the ranges reported by previous studies (28,36-40): $0.01-0.13 \mathrm{ppm}$ for the CN, 0.06-0.29 ppm for the GP, $0.02-0.14 \mathrm{ppm}$ for the PU, $-0.02-0.08 \mathrm{ppm}$ for the $\mathrm{TH}$, and 0.06-0.03 ppm for the PCR. In the StrS, only $\chi^{\text {NLFit }}$ had an average value close to the previously reported range for venous blood, namely, 0.17-0.58 ppm $(27,39,41,42)$ (Figure 3B).

Similar to the numerical phantom simulations, in the healthy volunteers, $\chi$ calculated using the NLFit pipeline $\left(\chi^{\text {NLFit }}\right)$ had slightly larger SDs in the GP, PU and StrS. In contrast with the numerical phantom simulations, in the healthy volunteers, $\chi$ calculated using the SNRwAvg pipeline $\left(\chi^{S N R-w A v g}\right)$ had slightly larger SDs in the CN, TH and PCR compared to $\chi^{\text {NLFit. }}$. 
Finally, in the numerical phantom simulations, the QSM image calculated using the NLFit pipeline had the largest RMSE value (108.8\%) followed, in decreasing order, by the QSM images calculated using the SNR-wAvg (94.7\%), TE-wAvg (94.0\%), Susc-wAvg (91.7\%) and Susc-TGV-wAvg pipelines (79.6\%) (Figure 2, bottom row).

\section{Phase noise propagation into the QSM images}

Figures 5 and 6 respectively show the estimated $\sigma(\chi)$ profiles in the numerical phantom simulations and a representative healthy subject. In both the phantom and the healthy volunteers, the NLFit and SNR-wAvg pipelines resulted in $\sigma(\chi)$ images with the lowest number of streaking artifacts (Figures 5A, E, C, G and 6A, E, C, G). Although a certain degree of streaking artifacts could be seen for all four pipelines, especially in the healthy volunteer the TE-wAvg and Susc-wAvg pipelines resulted in considerably worse streaking artifacts around high- $\chi$ venous structures.

\section{Statistical analysis}

In the healthy volunteers, for all processing pipelines and ROls, the Shapiro-Wilk test always rejected the hypothesis of normally distributed paired differences of $\chi$. Therefore, pairwise comparisons between pipelines were always evaluated using the nonparametric sign test. Significant differences between pipelines are highlighted in Figure 3B, whereas the betweenpipeline biases are shown in Figure 7. Biases smaller than $|0.01| \mathrm{ppm}(|\cdot|$ denotes the absolute value) were considered negligible.

A bias greater than $10.01 \mid$ ppm was observed for the NLFit pipeline relative to all other pipelines in the CN, GP and StrS, for the Susc-TGV-wAvg pipeline relative to the TE-wAvg, SNR-wAvg and Susc-wAvg pipelines in the GP, and between the SNR-wAvg and SuscwAvg pipelines in the GP (Figure 7).

In general, the bias was greater than $|0.01|$ ppm for the same pairs of pipelines that differed according to the sign test (Figures $3 \mathrm{~B}$ and 7 ).

\section{Discussion}

This study aimed to compare multi-echo combination methods for QSM, which were applied at different stages of the QSM processing pipeline before or after LBMs for phase unwrapping or background field removal. Methods for multi-echo combination were compared by applying each pipeline to numerically simulated data and images from healthy volunteers.

The relative accuracy of different processing pipelines in vivo was similar to the phantom simulations in the CN, GP and the venous ROls, where the NLFit pipeline provided the highest and most accurate means of $\chi$ (Figure 3 ). Slightly different relative accuracies were 
observed in the PU, TH and PCR. Here, however, no significant difference or systematic bias was detected between processing pipelines (Figures 3 and 7). Two exceptions were observed for the Susc-TGV-wAvg pipeline in the GP and the StrS, as this pipeline had the second highest mean of $\chi$ in the phantom simulations but the lowest mean of $\chi$ in the healthy volunteers (Figure 3). Both the TGV reconstruction pipeline and shortcomings of the numerically simulated data could explain these discrepancies in the performance of the Susc-TGV-wAvg pipeline. The numerically simulated data were generated based on a digital phantom containing regions of piece-wise constant $\chi$ distribution. As previously observed (43), piece-wise constant geometries allow good recovery of the underlying $\chi$ distribution using TGV-based algorithms, because the piece-wise constant constraints exactly match the underlying $\chi$ distribution. However, in regions with flow, anisotropic $\chi$ distributions or microstructure, these numerical models are likely to depart from a realistic representation of the head's bulk $\chi$. Notably, the GP, contrary to the PU and CN, is traversed by multiple myelinated axons of the striato-pallido-nigral tract. In the GP in vivo, additional anisotropic $\chi$ contrast dependent on axonal orientation could explain the lower accuracy of the Susc-TGVwAvg pipeline compared to the phantom simulations, in which the GP had isotropic $\chi$ (44). As for the StrS, the lower accuracy of the Susc-TGV-wAvg pipeline compared to the phantom simulations could be due to the presence of flow in vivo and the absence of flow compensation in the acquisition sequence, jointly violating the assumption of a piece-wise constant venous $\chi$. To overcome this assumption, future studies could exploit a newly developed realistic head phantom for QSM, which also incorporates microstructural effects (45).

Generally, the higher accuracy of the NLFit pipeline suggests that, for QSM, combining the temporally unwrapped multi-echo phase before applying LBMs for spatial phase unwrapping or $\Delta B_{B g}$ removal is preferable to combining the TE-dependent LBM-processed phase by averaging. This suggestion is in apparent contrast with the higher RMSE associated to the NLFit pipeline in the phantom simulations (Figure 2). However, the RMSE jointly reflects systematic and random errors, as it measures the bias between the estimated $\chi$ and $\chi^{\text {True }}$ but also reflects the variability of the estimated $\chi$ relative to its average value (43). Thus, the RMSE must always be interpreted in combination with complementary measurements of bias and precision.

There are several potential explanations as to why LBMs applied before multi-echo combination reduce the overall accuracy of QSM. Firstly, in contrast with path-based phase unwrapping, Laplacian phase unwrapping is likely to also remove some $\Delta B_{B g}$ components from the input phase image (23). This effect might be explained by the periodicity constraint 
imposed by evaluating the inverse Laplacian operator in Fourier domain $(9,23)$ and by the practical effect of inverting the Laplacian kernel via TSVD with a minimal truncation threshold (i.e., $10^{-10}$ ), which acts as a mild high-pass filter (10). Thus, the consistently lower accuracy of $\chi$ calculated using the TE-wAvg processing pipeline was probably driven by the incorrect assumption that the TE-dependent LBM-unwrapped phase corresponded to the true unwrapped phase. LBMs for $\Delta B_{B g}$ removal also applied tSVD (with a larger truncation threshold) but here the high pass filtering effect was expected, as background fields are slowly varying. Finally, the similar accuracy of $\chi$ calculated using the TE-wAvg, SNR-wAvg and Susc-wAvg pipelines suggests a negligible difference between averaging the Laplacian unwrapped phase over TEs before (TE-wAvg pipeline) or after TE-dependent Laplacian $\Delta B_{B g}$ removal (SNR-wAvg and Susc-wAvg pipelines).

In the numerical phantom simulations, all processing pipelines resulted in higher SDs of $\chi$ compared to the ground-truth, suggesting that they all intrinsically amplified the noise in the multi-echo signal phase. This result is in line with the known noise amplification of ill-posed inverse problems. However, the estimated SDs of $\chi$ varied across pipelines, with the SuscTGV-wAvg pipeline generally having the lowest SDs in both the numerical simulations and healthy volunteers (Figure 3). This result was in agreement with a previous study showing that, for QSM, $L_{1}$-regularization (as in TGV) provides better noise suppression than $L_{2^{-}}$ regularization (e.g., Tikhonov regularization) (6).

In both numerical simulations and healthy volunteers, based on the line profiles traced on the noise maps and streaking artifact reduction, the NLFit pipeline had a better noise mitigation than the TE-wAvg, SNR-wAvg and Susc-wAvg pipelines (Figures 5 and 6). This result suggests that combining the temporally unwrapped multi-echo phase by nonlinear complex fitting, designed to account for noise in the complex signal (5), results in better noise management than combining the multi-echo phase by averaging. As previously shown (5), errors in the combined field map mainly result from both noise in the signal phase and phase unwrapping errors near high- $\chi$ regions (e.g., the veins). Both sources of error were successfully managed by nonlinear complex fitting, as shown by the dramatic reduction of streaking artifacts in Figure 6A. In line with previous observations (25), this result also suggests that the regularization strategy employed by the $\Delta B_{L o c}$-to $\chi$ step can mitigate artifactual streaking errors only when major sources of error in the field map have been tightly constrained. In both the numerical simulations and healthy volunteers, the SNR-wAvg pipeline had the best noise mitigation of all averaging-based pipelines (Figures 5 and 6). Thus, if applying nonlinear complex fitting is not possible, averaging using the SNRweighting method could offer the best alternative for noise reduction. 
All these indications do not necessarily apply to $\chi$ estimation in WM tissue. Indeed, due to WM's ordered microstructure, QSM of WM requires acquiring GRE images at multiple head orientations and modelling $\chi$ as a tensor $(46,47)$. Thus, further studies are needed to evaluate the translatability of these results to WM tissue.

In the present study, all experiments were limited to one field strength (i.e., 3 T). As tissue relaxation times (e.g., $T_{2}^{*}$ ) shorten with increasing field strength but the signal phase at a given TE increases, further work is needed to assess the translatability of these results to ultrahigh fields. Finally, it must be noted that in the numerical phantom simulations, all processing pipelines underestimated $\chi^{\text {True }}$ (Figure 3). However, in QSM, some degree of underestimation is always expected, because of the ill-posed nature of the $\Delta B_{\text {Loc }}$-to- $\chi$ inverse problem (16).

\section{Conclusions}

For QSM using multi-echo acquisitions, combining the multi-echo phase by nonlinear complex fitting before applying LBMs for phase unwrapping or $\Delta B_{B g}$ removal offers the best compromise between bias reduction and noise mitigation.

\section{Acknowledgments}

We thank all the healthy volunteers who participated in this study. We also thank Dr. Rosa Cortese and Dr. Floriana De Angelis (Queen Square MS Centre, UCL Queen Square Institute of Neurology, UCL, London, UK) for their help with the MRI scans. We are indebted to Prof. Claudia Gandini Wheeler-Kingshott (NMR Unit, Queen Square MS Centre, UCL Queen Square Institute of Neurology, UCL, London, UK) for her support with the MRI scans performed at the Queen Square MS Centre, UCL.

\section{References}

1. Wei H, Dibb R, Zhou Y, Sun Y, Xu J, Wang N, Liu C. Streaking artifact reduction for quantitative susceptibility mapping of sources with large dynamic range. NMR Biomed 2015;28(10):1294-1303.

2. Li W, Wang N, Yu F, Han H, Cao W, Romero R, Tantiwongkosi B, Duong TQ, Liu C. A method for estimating and removing streaking artifacts in quantitative susceptibility mapping. Neuroimage 2015;108:111-122.

3. Wu B, Li W, Avram AV, Gho SM, Liu C. Fast and tissue-optimized mapping of magnetic susceptibility and T2* with multi-echo and multi-shot spirals. Neuroimage 2012;59(1):297-305.

4. Chen Y, Liu S, Wang Y, Kang Y, Haacke EM. STrategically Acquired Gradient Echo (STAGE) imaging, part I: Creating enhanced T1 contrast and standardized 
susceptibility weighted imaging and quantitative susceptibility mapping. Magn Reson Imaging 2018;46:130-139.

5. Liu T, Wisnieff C, Lou M, Chen W, Spincemaille P, Wang Y. Nonlinear formulation of the magnetic field to source relationship for robust quantitative susceptibility mapping. Magn Reson Med 2013;69(2):467-476.

6. Kressler B, de Rochefort L, Liu T, Spincemaille P, Jiang Q, Wang Y. Nonlinear regularization for per voxel estimation of magnetic susceptibility distributions from MRI field maps. IEEE Trans Med Imaging 2010;29(2):273-281.

7. de Rochefort L, Brown R, Prince MR, Wang Y. Quantitative MR susceptibility mapping using piece-wise constant regularized inversion of the magnetic field. Magn Reson Med 2008;60(4):1003-1009.

8. Robinson SD, Bredies K, Khabipova D, Dymerska B, Marques JP, Schweser F. An illustrated comparison of processing methods for MR phase imaging and QSM: combining array coil signals and phase unwrapping. NMR Biomed 2017;30(4).

9. Schofield MA, Zhu Y. Fast phase unwrapping algorithm for interferometric applications. Opt Lett 2003;28(14):1194-1196.

10. Schweser F, Robinson SD, de Rochefort L, Li W, Bredies K. An illustrated comparison of processing methods for phase MRI and QSM: removal of background field contributions from sources outside the region of interest. NMR Biomed 2017;30(4).

11. Biondetti E, Karsa A, Thomas DL, Shmueli K. Investigating the accuracy and precision of TE-dependent versus multi-echo QSM using Laplacian-based methods at 3 T. Magn Reson Med 2020.

12. Qiu D, Brown RC, Sun B, Palasis S, Burns TG, Jones RA. Abnormal Iron Levels in the Brain of Pediatric Sickle Cell Disease Patients: a Study using Quantitative Susceptibility Mapping (QSM). Proceedings of the International Society for Magnetic Resonance in Medicine 2014;22:897.

13. Langkammer C, Bredies K, Poser BA, Barth M, Reishofer G, Fan AP, Bilgic B, Fazekas F, Mainero C, Ropele S. Fast quantitative susceptibility mapping using 3D EPI and total generalized variation. Neuroimage 2015;111:622-630.

14. Conturo TE, Smith GD. Signal-to-noise in phase angle reconstruction: Dynamic range extension using phase reference offsets. Magnetic Resonance in Medicine 1990;15(3):420-437.

15. Liu T, Khalidov I, de Rochefort L, Spincemaille P, Liu J, Tsiouris AJ, Wang Y. A novel background field removal method for MRI using projection onto dipole fields (PDF). NMR Biomed 2011;24(9):1129-1136. 
16. Wang Y, Liu T. Quantitative susceptibility mapping (QSM): Decoding MRI data for a tissue magnetic biomarker. Magn Reson Med 2015;73(1):82-101.

17. Shmueli K, de Zwart JA, van Gelderen P, Li TQ, Dodd SJ, Duyn JH. Magnetic susceptibility mapping of brain tissue in vivo using MRI phase data. Magn Reson Med 2009;62(6):1510-1522.

18. Biondetti E, Thomas DL, Shmueli K. Application of Laplacian-based Methods to Multi-echo Phase Data for Accurate Susceptibility Mapping. Proceedings of the International Society for Magnetic Resonance in Medicine 2016;24:1547.

19. Pruessmann KP, Weiger M, Scheidegger MB, Boesiger P. SENSE: Sensitivity encoding for fast MRI. Magnetic Resonance in Medicine 1999;42(5):952-962.

20. Zubal IG, Harrell CR, Smith EO, Smith AL, Krischlunas P. Two dedicated software, voxel-based, anthropomorphic (torso and head) phantoms. Proceeding of the International Conference at the National Radiological Protection Board1995. p 105111.

21. Jenkinson M, Beckmann CF, Behrens TE, Woolrich MW, Smith SM. Fsl. Neuroimage 2012;62(2):782-790.

22. Smith SM. Fast robust automated brain extraction. Hum Brain Mapp 2002;17(3):143155.

23. Schweser F, Deistung A, Sommer K, Reichenbach JR. Toward online reconstruction of quantitative susceptibility maps: superfast dipole inversion. Magn Reson Med 2013;69(6):1582-1594.

24. Wu B, Li W, Guidon A, Liu C. Whole brain susceptibility mapping using compressed sensing. Magn Reson Med 2012;67(1):137-147.

25. Acosta-Cabronero J, Milovic C, Mattern H, Tejos C, Speck O, Callaghan MF. A robust multi-scale approach to quantitative susceptibility mapping. Neuroimage 2018;183:7-24.

26. Hansen PC, O'Leary DP. The Use of the L-Curve in the Regularization of Discrete IIIPosed Problems. SIAM Journal on Scientific Computing 1993;14(6):1487-1503.

27. Biondetti E, Rojas-Villabona A, Sokolska M, Pizzini FB, Jager HR, Thomas DL, Shmueli K. Investigating the oxygenation of brain arteriovenous malformations using quantitative susceptibility mapping. Neuroimage 2019;199:440-453.

28. Lim IA, Faria AV, Li X, Hsu JT, Airan RD, Mori S, van Zijl PC. Human brain atlas for automated region of interest selection in quantitative susceptibility mapping: application to determine iron content in deep gray matter structures. Neuroimage 2013;82:449-469. 
29. Modat M, Ridgway GR, Taylor ZA, Lehmann M, Barnes J, Hawkes DJ, Fox NC, Ourselin S. Fast free-form deformation using graphics processing units. Comput Methods Programs Biomed 2010;98(3):278-284.

30. Ourselin S, Roche A, Subsol G, Pennec X, Ayache N. Reconstructing a 3D structure from serial histological sections. Image and Vision Computing 2001;19(1-2):25-31.

31. Yushkevich PA, Piven J, Hazlett HC, Smith RG, Ho S, Gee JC, Gerig G. User-guided $3 D$ active contour segmentation of anatomical structures: significantly improved efficiency and reliability. Neuroimage 2006;31(3):1116-1128.

32. Lerski RA, de Wilde J, Boyce D, Ridgway J. Quality control in magnetic resonance imaging. York, UK: The Institute of Physics and Engineering in Medicine; 1998.

33. Dietrich O, Raya JG, Reeder SB, Reiser MF, Schoenberg SO. Measurement of signal-to-noise ratios in MR images: influence of multichannel coils, parallel imaging, and reconstruction filters. J Magn Reson Imaging 2007;26(2):375-385.

34. Weiger M, Pruessmann KP, Boesiger P. 2D SENSE for faster 3D MRI. MAGMA 2002;14(1):10-19.

35. Gudbjartsson H, Patz S. The Rician distribution of noisy MRI data. Magn Reson Med 1995;34(6):910-914.

36. Li W, Wu B, Liu C. Quantitative susceptibility mapping of human brain reflects spatial variation in tissue composition. Neuroimage 2011;55(4):1645-1656.

37. Wharton $\mathrm{S}$, Bowtell R. Whole-brain susceptibility mapping at high field: a comparison of multiple- and single-orientation methods. Neuroimage 2010;53(2):515-525.

38. Bilgic B, Pfefferbaum A, Rohlfing T, Sullivan EV, Adalsteinsson E. MRI estimates of brain iron concentration in normal aging using quantitative susceptibility mapping. Neuroimage 2012;59(3):2625-2635.

39. Deistung A, Schäfer A, Schweser F, Biedermann U, Turner R, Reichenbach JR. Toward in vivo histology: A comparison of quantitative susceptibility mapping (QSM) with magnitude-, phase-, and R2*-imaging at ultra-high magnetic field strength. Neurolmage 2013;65:299-314.

40. Schweser F, Deistung A, Lehr BW, Reichenbach JR. Quantitative imaging of intrinsic magnetic tissue properties using MRI signal phase: an approach to in vivo brain iron metabolism? Neuroimage 2011;54(4):2789-2807.

41. Fan AP, Bilgic B, Gagnon L, Witzel T, Bhat H, Rosen BR, Adalsteinsson E. Quantitative oxygenation venography from MRI phase. Magn Reson Med 2014;72(1):149-159. 
42. Cetin S, Bilgic B, Fan A, Holdsworth S, Unal G. Vessel Orientation Constrained Quantitative Susceptibility Mapping (QSM) Reconstruction. Ourselin S, Joskowicz L, Sabuncu MR, Unal G, Wells W, editors. Cham: Springer International Publishing; 2016.

43. Langkammer C, Schweser F, Shmueli K, Kames C, Li X, Guo L, Milovic C, Kim J, Wei H, Bredies K, Buch S, Guo Y, Liu Z, Meineke J, Rauscher A, Marques JP, Bilgic B. Quantitative susceptibility mapping: Report from the 2016 reconstruction challenge. Magn Reson Med 2018;79(3):1661-1673.

44. Duyn JH, Schenck J. Contributions to magnetic susceptibility of brain tissue. NMR Biomed 2017;30(4).

45. Marques JP, Meineke J, Milovic C, Bilgic B, Chan KS, Hedouin R, van der Zwaag W, Langkammer C, Schweser F. QSM reconstruction challenge 2.0: A realistic in silico head phantom for MRI data simulation and evaluation of susceptibility mapping procedures. Magn Reson Med 2021;86(1):526-542.

46. Li W, Liu C, Duong TQ, van Zijl PC, Li X. Susceptibility tensor imaging (STI) of the brain. NMR Biomed 2017;30(4).

47. Chen WC, Foxley S, Miller KL. Detecting microstructural properties of white matter based on compartmentalization of magnetic susceptibility. Neuroimage 2013;70:1-9. 


\section{Figures}

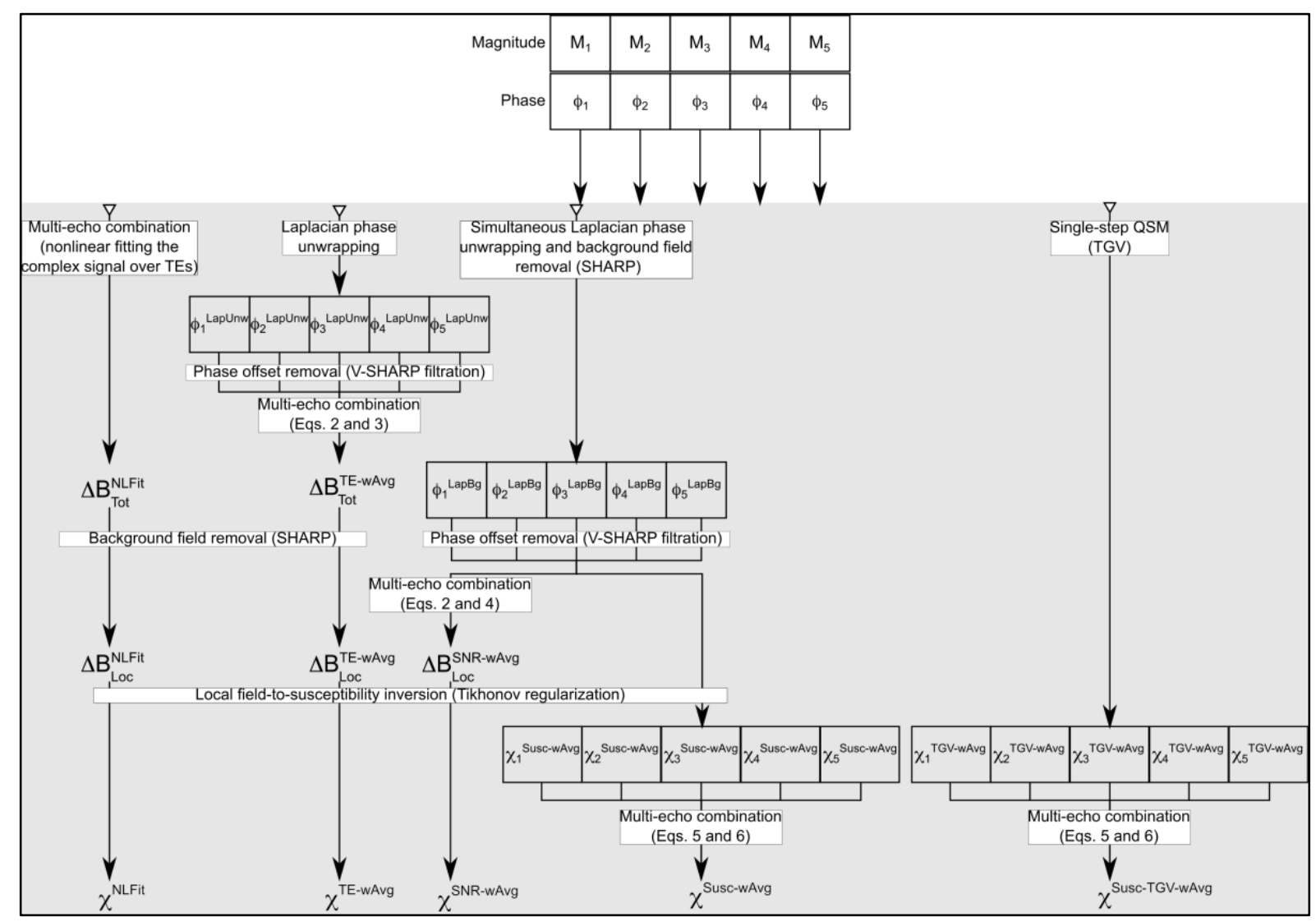

Figure 1. Processing pipelines for multi-echo QSM. For each multi-echo combination method (NLFit [nonlinear phase fitting], TE-wAvg [TE-weighted phase averaging], SNRwAvg [SNR-weighted phase averaging], Susc-wAvg and Susc-TGV-wAvg [magnitudeweighted susceptibility averaging]) the processing steps are described as separate processing streams in the grey box. 


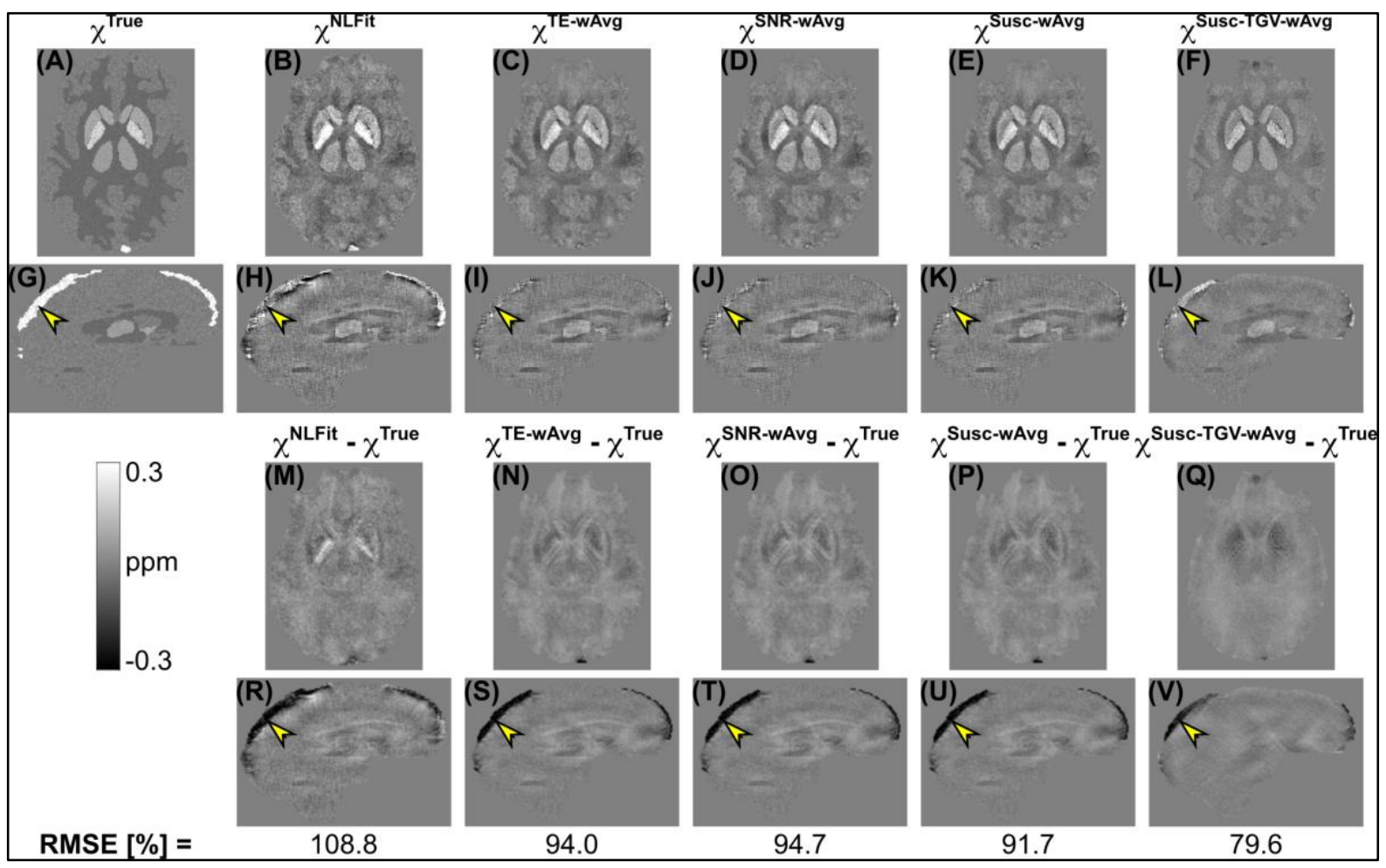

Figure 2. QSM images calculated using distinct multi-echo combination methods in

the numerical phantom simulations. In the numerical phantom, the same transverse and sagittal slices are shown for the ground-truth susceptibility map $(A, G)$, and for the susceptibility maps calculated using NLFit $(B, H)$, TE-wAvg (C, I), SNR-wAvg (D, J), SuscwAvg $(E, K)$ and Susc-TGV-wAvg $(F, L)$. The figure also shows the difference between each susceptibility map and the ground truth $(\mathrm{M}-\mathrm{V})$. The bottom row shows the root mean squared errors (RMSEs) of $\chi$. In all the sagittal images (G-L, R-V), the yellow arrowheads point at the same location in the SSS. 


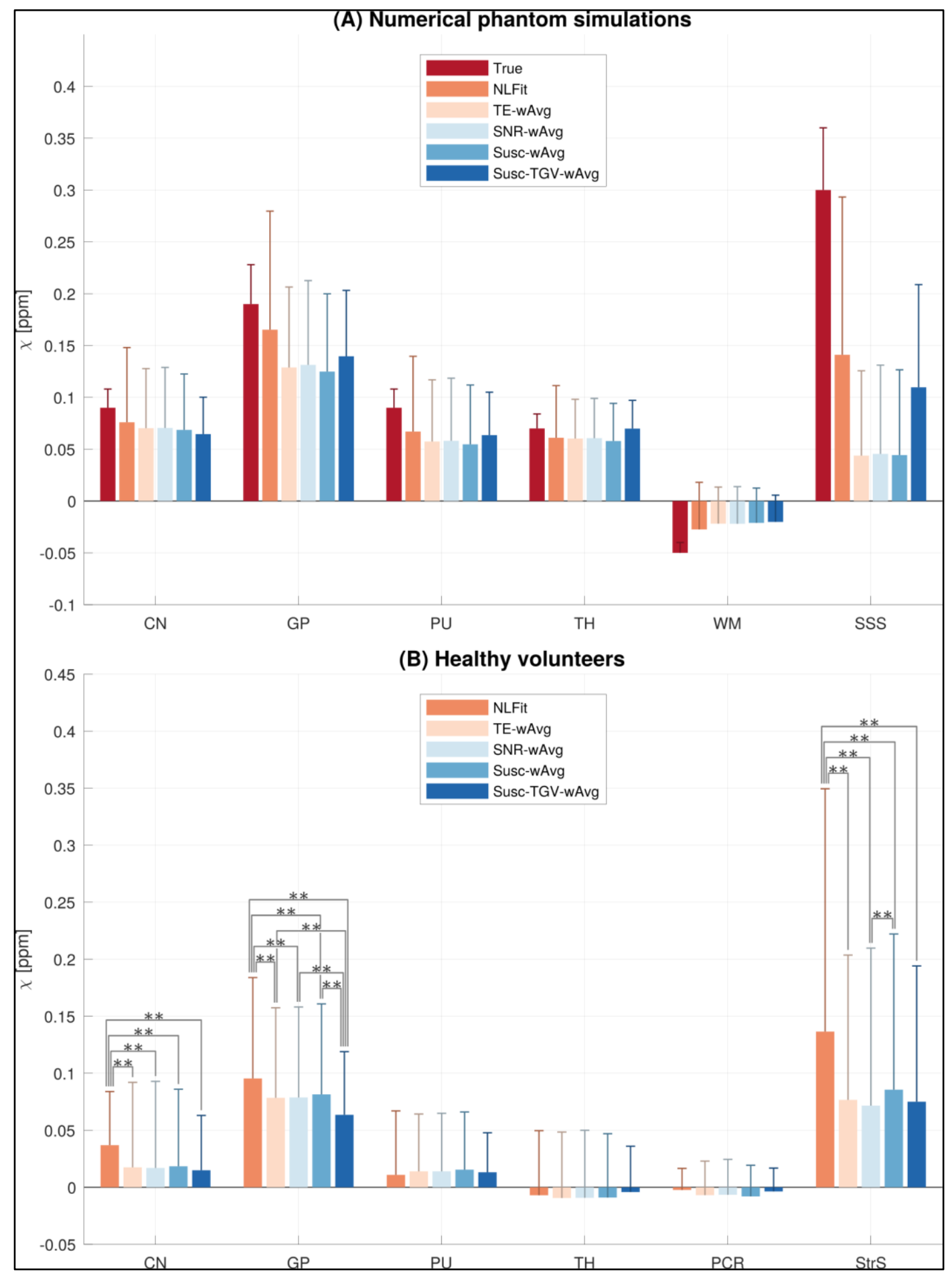

Figure 3. Means and SDs of $\chi$ in the phantom and healthy volunteer ROls. The means and SDs (error bars) of $\chi$ are shown in each ROI of the numerically simulated $(\mathrm{A})$ and pooled healthy volunteer data $(B)$ for each processing pipeline. In the numerical phantom the ground-truth $\chi$ is also shown. In the healthy volunteers, significant differences between pairs of pipelines are denoted using the symbol ${ }^{* *}(P$-value $<0.05)$. Abbreviations: $\mathrm{CN}$, caudate nucleus; GP, globus pallidus; PCR, posterior corona radiata; PU, putamen; StrS, straight sinus; $\mathrm{TH}$, thalamus; WM, white matter. 


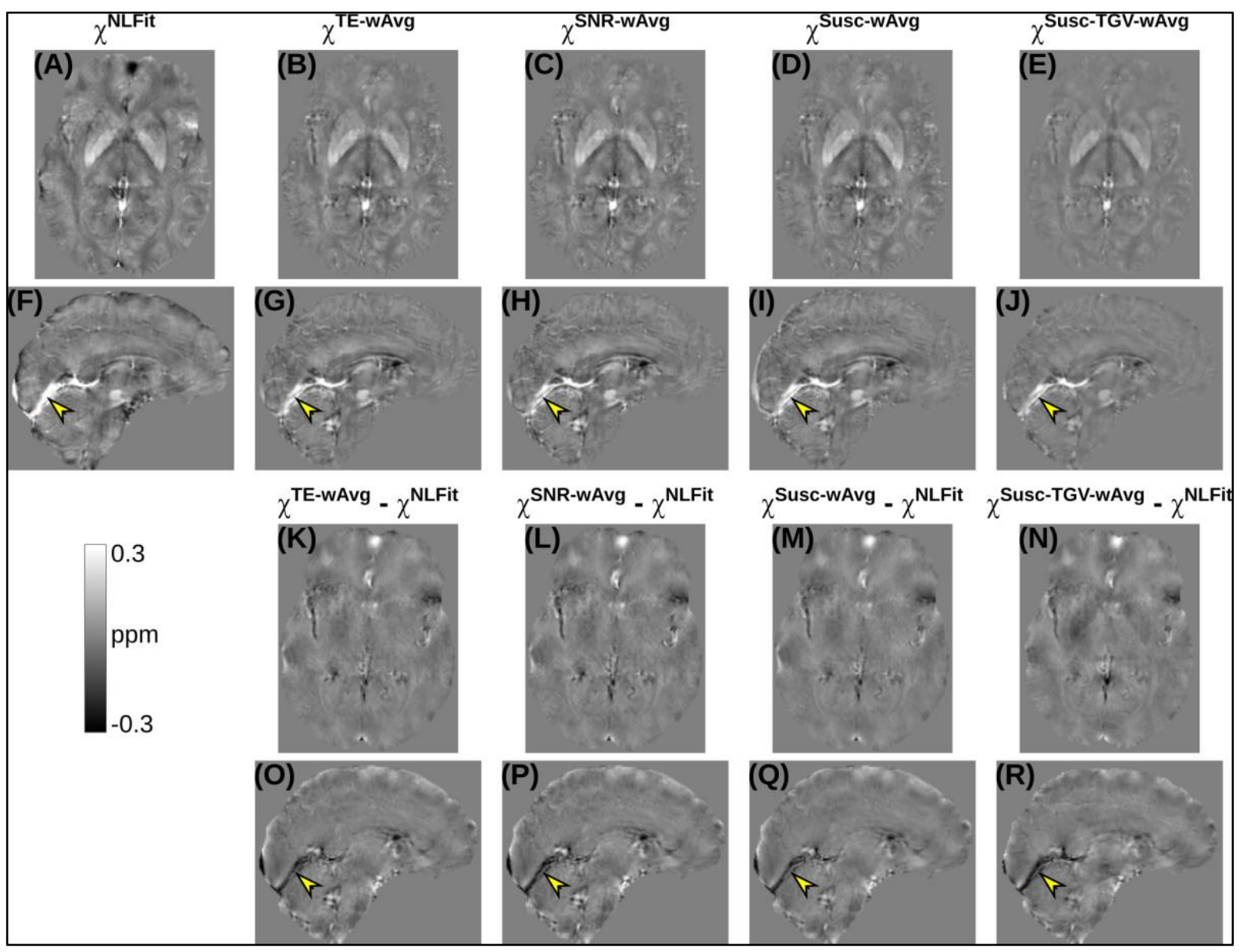

Figure 4. QSM images calculated using distinct multi-echo combination methods in a

representative healthy volunteer. In a representative healthy volunteer, the same transverse and sagittal slices are shown for the susceptibility maps calculated using NLFit $(A, F)$ [nonlinear phase fitting], TE-wAvg $(B, G)$ [TE-weighted phase averaging], SNR-wAvg $(C, H)$ [SNR-weighted phase averaging], Susc-wAvg (D, I) and Susc-TGV-wAvg (E, J) [magnitude-weighted susceptibility averaging]. The figure also shows the differences between the TE-wAvg, SNR-wAvg, Susc-wAvg and Susc-TGV-wAvg maps and the NLFit map $(K-R)$. In all the sagittal images (F-J, O-R), the yellow arrowheads point at the same location in the StrS. 


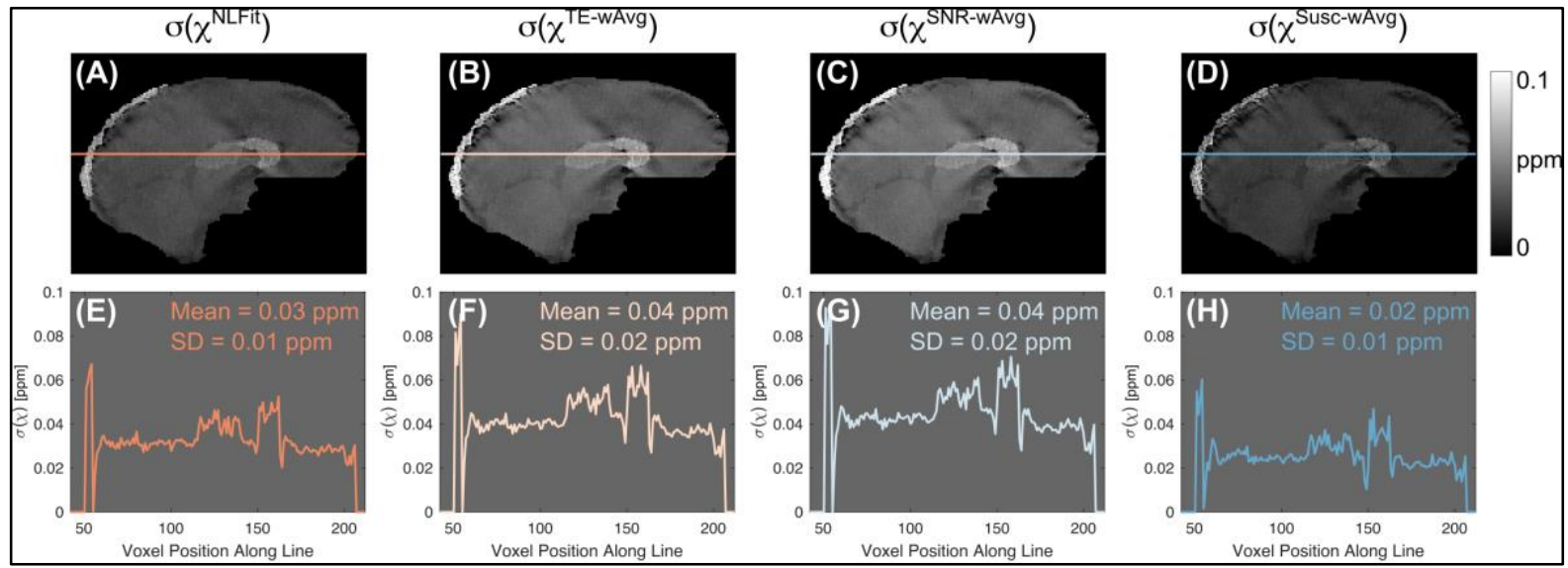

Figure 5. Susceptibility noise profiles in the numerical phantom simulations. In the numerical phantom, the same sagittal slice is shown for susceptibility noise $(\sigma(\chi))$ images calculated using the NLFit, TE-wAvg, SNR-wAvg and Susc-wAvg pipelines (A-D). The susceptibility noise is plotted for a line profile traced on the $\sigma(\chi)$ images $(E-H)$ including the high- $\chi$ SSS.

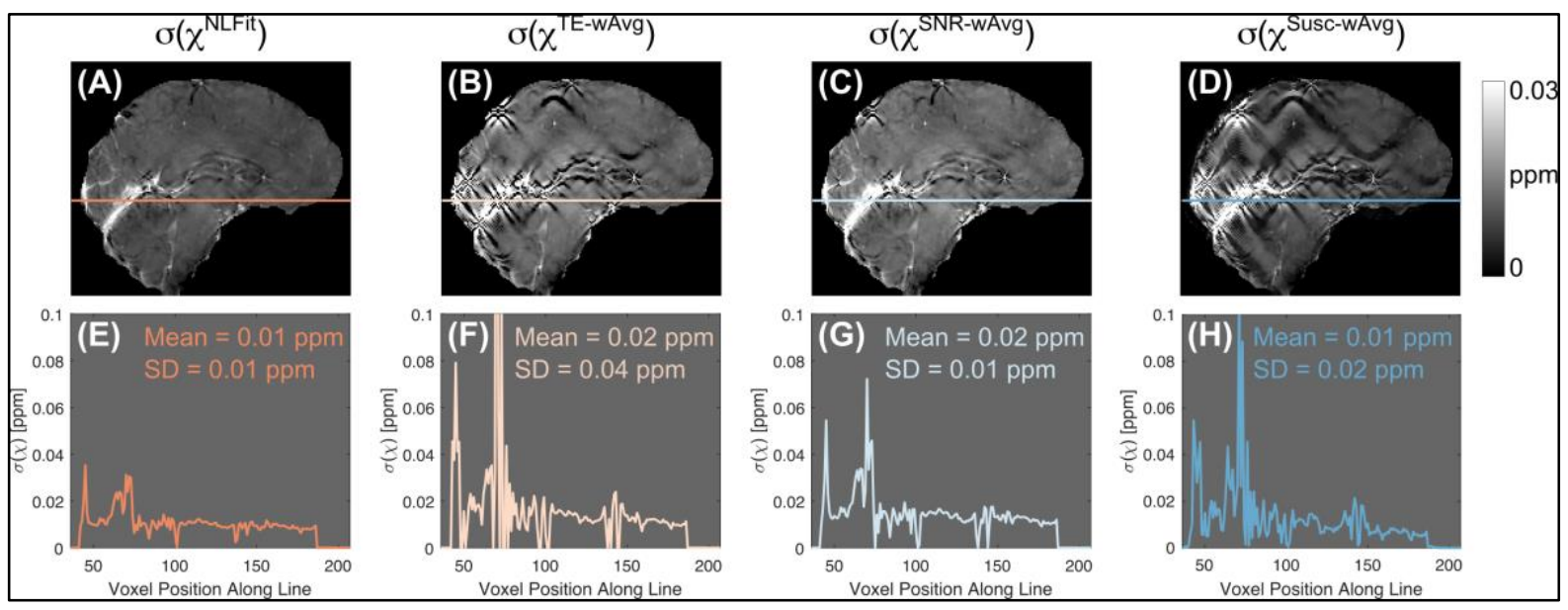

Figure 6. Susceptibility noise profiles in a representative healthy volunteer. For a representative healthy volunteer, the same sagittal slice is shown for susceptibility noise $(\sigma(\chi))$ images calculated using the NLFit, TE-wAvg, SNR-wAvg and Susc-wAvg pipelines (A-D). The susceptibility noise is plotted for a line profile traced on the $\sigma(\chi)$ images $(E-H)$ including the high- $\chi$ StrS. 


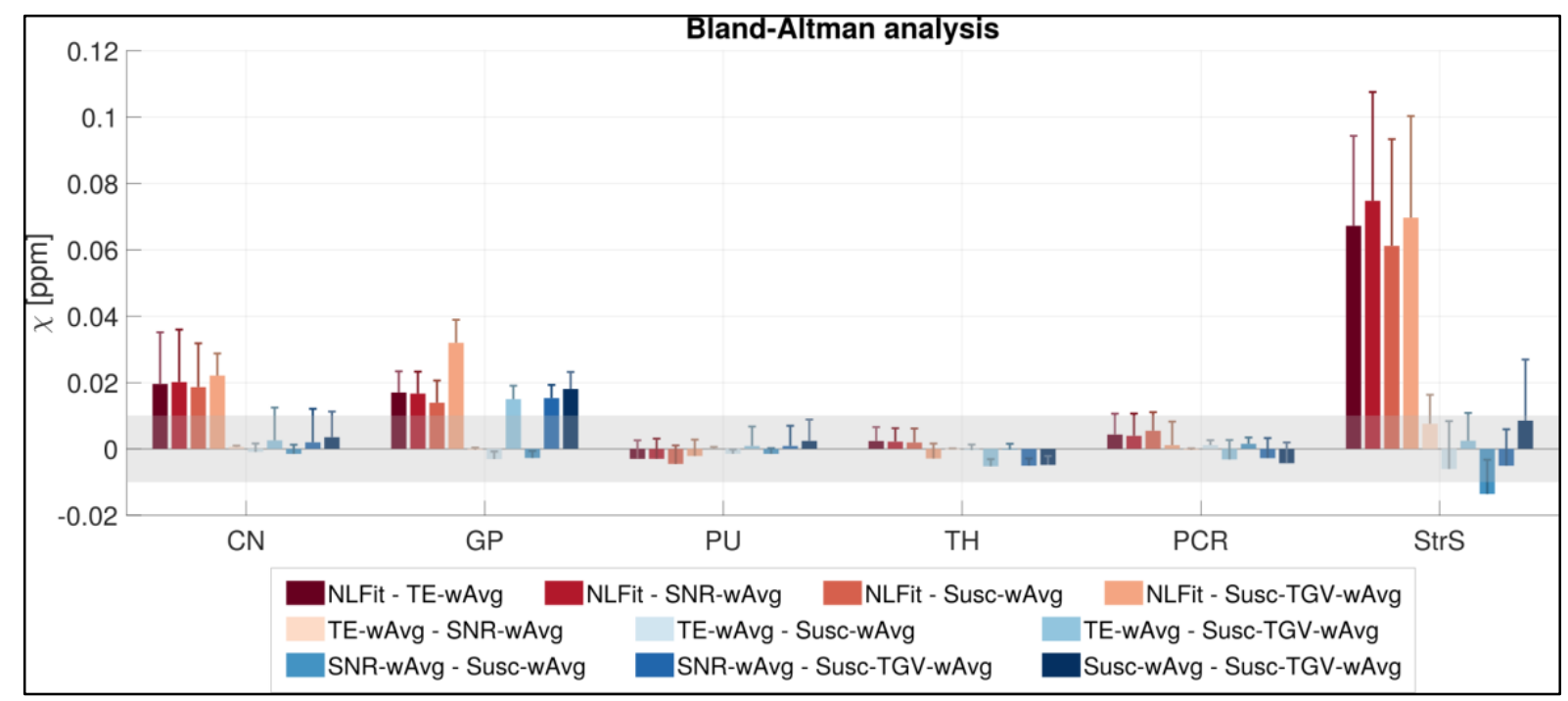

Figure 7. Bias between multi-echo pipelines for QSM in each ROI. The mean and SDs (error bars) of the bias are shown in each healthy volunteer ROI for all pairs of multi-echo processing pipelines. The grey band denotes the $[-0.01-0.01] \mathrm{ppm}$ interval. If the mean of the bias was within this interval, the difference between the corresponding pair of QSM pipelines was considered negligible. 